\title{
COMPETENCIA MOTRIZ EN ESCOLARES DE PRIMER Y SEGUNDO AÑO DE PRIMARIA EN LA REGIÓN DE ARAUCANÍA, CHILE
}

\section{MOTOR COMPETENCE IN SCHOOLCHILDREN OF FIRST AND SECOND GRADE IN THE ARAUCANÍA REGION, CHILE}

\author{
Nicolas Martinez-Lopez ${ }^{1}$, Miguel Espinoza-Silva ${ }^{1}$, Jaime Carcamo-Oyarzun ${ }^{1}$ \\ n.martinez11@ufromail.cl; jose.espinoza@ufrontera.cl; jaime.carcamo@ufrontera.cl
}

${ }^{1}$ Universidad de La Frontera, Temuco, Chile

Envío original: 2021-01-29 Reenviado: 2021-06-10 Aceptado: 2021-08-20

Publicado: 2021-10-21

Doi: https://doi.org/10.15517/pensarmov.v19i2.45621

\begin{abstract}
RESUMEN
La competencia motriz es un componente importante para la participación en la cultura del juego, deportes y actividad física, por lo que su desarrollo es fundamental en el inicio de la trayectoria escolar. Este estudio tuvo como propósitos describir el desempeño en la competencia motriz de Control de Objetos y Control del Cuerpo de estudiantes de primer y segundo grado de primaria de Chile; determinar la relación entre la competencia motriz y la edad; y establecer la existencia de posibles diferencias según el sexo de los estudiantes. Se utilizó un enfoque cuantitativo, siendo un estudio no experimental de corte transversal y de tipo descriptivo-correlacional. Participaron 289 escolares (51,2\% niñas, 48,8\% niños) de entre seis y ocho años de cinco establecimientos educacionales de la región de La Araucanía, Chile. Se utilizó el test MOBAK 1-2 para la evaluación de la competencia motriz en dos dimensiones (Control de Objetos y Control del Cuerpo). Para establecer la relación entre competencia motriz y edad se utilizó el coeficiente rho de Spearman, y para determinar la existencia de diferencias según sexo se utilizó la prueba $U$ de Mann-Whitney, determinando el tamaño del efecto a través del cálculo de probabilidad de superioridad (PS). Los resultados indican que la edad se relaciona de forma positiva con el Control de Objetos $(p<0.001$, $r=0.315$ ) y el Control del Cuerpo $(p<0.001, r=0.261)$. Al comparar los desempeños motrices según sexo, los niños presentan mejores resultados que las niñas en tareas motrices asociadas al Control de Objetos $\left(U=13533.000, Z=4.439, p<0.001, P S_{\text {est }}=0.61\right)$, mientras que en Control del Cuerpo no se encontraron diferencias estadísticamente significativas. Tanto la edad como el sexo de los estudiantes son variables asociadas al desarrollo de la competencia
\end{abstract}


motriz. Estos resultados aportan información relevante para diseñar estrategias pedagógicas que busquen fomentar la competencia motriz de manera óptima y equitativa.

Palabras clave: habilidades motrices en niños, evaluación de habilidades motrices, educación física.

\section{ABSTRACT}

Motor competence is an important factor for the participation in the culture of play, sports and physical activity, so their development during the first years of school is essential. The purpose of this study was to describe performances in the motor competencies Object Control and Self-movement of first and second grade students, to establish the relationship between motor competence and the students' age, and to determinate possible differences according to gender. A quantitative approach was used, being a non-experimental, cross-sectional, descriptive-correlational study. A total of 289 schoolchildren (51.2\% girls, $48.8 \%$ boys) between the ages of six and eight of five schools from the La Araucanía Region, Chile, participated. The MOBAK 1-2 test was used to evaluate motor competence in two dimensions (Object Control and Self-movement). To establish the relationship between motor competence and age, Spearman's rho coefficient was used, and to determine the existence of differences according to gender, the Mann-Whitney $U$ test was used, determining the effect size by calculating the probability of superiority (PS). The results indicate that the age is positively related to Object Control $(p<0.001, r=0.315)$ and Self-movement $(p<0.001, r=0.261)$. Comparing the motor performance by gender, the boys showed better results than the girls in motor tasks associated with Object Control $\left(U=13533.000, Z=4.439, p<0.001, P_{\text {est }}=0.61\right)$, while no statistically significant differences were found in the dimension of Self-movement. Both age and gender of students are variables associated with the development of motor competence. These results provide relevant information that should be considered in the design of pedagogical strategies that seek to promote an optimal and equitable development of motor competence.

Key words: motor ability in children, motor ability test, physical education.

\section{INTRODUCCIÓN}

El desarrollo de la competencia motriz en la etapa de la niñez es fundamental para la formación integral de las personas, por lo que es considerada como uno de los objetivos fundamentales de las clases de Educación Física, ya que favorece la participación de los estudiantes en situaciones de juego, deporte y actividad física (Herrmann, Heim, y Seelig, 2019). Producto de esta relevancia, el estudio de la competencia motriz ha generado gran 
interés en el último tiempo, convirtiéndose en un tópico de investigación recurrente, no tan solo en el ámbito de la Educación Física o del desarrollo motor, sino que también en el área de la actividad física y salud (Lopes et al, 2021). Desde que Stodden et al. (2008) plantearon un modelo conceptual sobre la dinámica y sinergia del rol que la competencia motriz cumple en la iniciación, mantenimiento o abandono de la actividad física, diversas investigaciones han confirmado el potencial de la competencia motriz en la promoción de trayectorias positivas o negativas para la práctica de actividad física y la salud (Robinson et al., 2015). Revisiones sistemáticas han planteado la existencia de asociaciones entre los niveles de competencia motriz en la niñez y el incremento de la actividad física en estadios posteriores, sin embargo, aún no se ha determinado cómo se producirían estas relaciones (Holfelder y Schott, 2014; Logan, Kipling Webster, Getchell, Pfeiffer, y Robinson, 2015). Asimismo, se ha planteado que un correcto desarrollo de la competencia motriz se asocia con los niveles de actividad física en la adultez, por lo que debe ser considerada como una de las determinantes para la adherencia de la práctica de actividad física y alfabetización motriz a lo largo de la vida (Cairney, Dudley, Kwan, Bulten, y Kriellaars, 2019).

Si bien es cierto que este interés emergente se ha relacionado con el fomento a la adherencia de la práctica de actividad física, el desarrollo de la competencia motriz en las clases de Educación Física tiene una data mayor. Ya en $\underline{1995}$, Ruiz indicaba que la competencia motriz hace referencia al

conjunto de conocimientos, procedimientos, actitudes y sentimientos que intervienen en las múltiples interacciones que realiza en su medio y con los demás, y que permiten que los escolares superen exitosamente los diferentes problemas motrices planteados, tanto en las sesiones de educación física como en su vida cotidiana. (Ruiz Perez, 1995, p. 19).

De esta definición se desprende que la competencia motriz no solo se circunscribe al ámbito físico, sino que considera una mirada global de cómo lo cognitivo, motor, social y emocional se entremezclan de forma intensa (Ruiz Perez, 1995). En esta misma línea, Gerlach, Herrmann, Jekauc, y Wagner (2017) plantean que la competencia motriz debe ser entendida como un desempeño funcional latente, que se desarrolla al enfrentar demandas motrices específicas, lo que implica aspectos biológicos, pero también emocionales y motivacionales. Al ser un desempeño funcional latente, la competencia motriz no es observable directamente, sino que se evidencia a través de la resolución de tareas motrices por medio de las habilidades motrices (Gerlach et al., 2017). Las habilidades motrices se definen como los "bloques de construcción" de movimientos más complejos y necesarios para participar en deportes, juegos u otra actividad física específica (Gallahue, Ozmun, y Goodway, 2012; Logan, Ross, Chee, Stodden, y Robinson,_2018). Estas habilidades han sido 
clasificadas de diversas maneras según el enfoque dado por diferentes autores, aunque en el fondo dichas clasificaciones son muy similares. Tradicionalmente se han catalogado en habilidades de locomoción (como correr, saltar, galopar); habilidades de control de objetos (como lanzar, manipular, atrapar, patear); y habilidades de estabilidad, (como equilibrarse o balancearse) (Gallahue et al., 2012). Desde una perspectiva del desarrollo motor a lo largo de la vida, las habilidades motrices también han sido diferenciadas en habilidades de locomoción (que también consideran tareas como correr, saltar, galopar y desplazarse de forma lateral); habilidades balísticas (como lanzar, patear y batear); y habilidades de manipulación (como agarrar, atrapar, alcanzar) (Haywood y Getchell, 2019). Considerando también la perspectiva de la evaluación de estas habilidades motrices, dependiendo de la batería de test, se han clasificado en control de objetos (actividades como lanzar, atrapar, batear, patear) y locomoción (como correr, galopar, saltar) (Ulrich, 1985), o en control de objetos (como lanzar, atrapar, conducir un balón) y control del cuerpo (como rodar, saltar, equilibrarse) (Herrmann et al., 2019). Considerando la naturaleza de estas categorías, la evaluación de cada una de ellas se realiza de manera independiente, es decir, diferenciándose el desempeño de la manipulación o control de objetos y la de locomoción o control del cuerpo (Herrmann, 2018; Ulrich, 1985), aunque también es posible encontrar reportes que contrastan ambas categorías (Foulkes et al., 2015).

El desarrollo de la competencia motriz está determinado por diferentes factores, dentro de los cuales destacan la edad y el sexo. En lo que respecta a la variable edad, Barnett et al. (2016), en una revisión sistemática y metaanálisis sobre los correlatos de la competencia motriz, identificaron que el incremento de la edad se relaciona de forma positiva con la competencia motriz; sin embargo, esta evidencia no sería absoluta, ya que otros estudios no han encontrado asociaciones entre la edad y el desempeño motriz (Jiménez-Díaz, SalazarRojas, y Morera, 2015), sobre todo en la proporción de estudiantes que presentan bajos niveles de competencia motriz (Hardy, Reinten-Reynolds, Espinel, Zask y Okely, 2012). En cuanto a la variable sexo, el rol que juega como factor determinante no está del todo consensuado, ya que existen diferentes asociaciones según el tipo de tarea motriz. En lo que se refiere a las tareas motrices vinculadas al control de objetos, la mayoría de los estudios indican que los niños serían más competentes que las niñas (Barnett et al., 2016; livonen y Sääkslahti, 2014); mientras que en las tareas relacionadas con el control del cuerpo y estabilidad los estudios presentan resultados heterogéneos, ya que en algunos se ha indicado que las niñas presentarían un mejor desempeño que los niños (livonen y Sääkslahti, 2014), sin embargo, en otras investigaciones no se han encontrado asociaciones en función del sexo (Barnett et al., 2016).

El desarrollo de la competencia motriz no se produce de forma automática, sino que necesita ser enseñada, por lo que todos los estudiantes cuentan con el potencial de 
desarrollar y aprender patrones de movimiento fundamentales que permitirán adquirir habilidades motrices más especializadas (Morgan et al., 2013). Es aquí en donde la Educación Física cumple un rol relevante en el fomento de la competencia motriz (Ennis, 2011), ya que las prácticas pedagógicas utilizadas por el profesorado en las clases de Educación Física se consideran determinantes críticos del desarrollo de niveles óptimos de desempeño motriz (Dyson, 2014). En consecuencia, su evaluación es de suma importancia para diagnosticar y monitorear los resultados del aprendizaje (Scheuer, Herrmann, y Bund, 2019), por lo que el profesorado de Educación Física debe considerar la evaluación constante de la competencia motriz, tanto para detectar problemas de desarrollo de las habilidades motrices, así como para monitorear el logro de los aprendizajes, para que de esta forma las estrategias pedagógicas se orienten según las necesidades de los estudiantes (Quitério et al., 2018). A pesar de que el currículum chileno de Educación Física considera a las habilidades motrices como el eje fundamental de la asignatura (Ministerio de Educación, 2013); no se explicita la evaluación de los objetivos vinculados a ella, quedando a criterio del docente, quien no cuenta con parámetros locales de diagnóstico ya que existe muy poca evidencia del desempeño motriz de la población escolar chilena (Pavez-Adasme et al.,2020). Ante esta necesidad, el presente estudio tiene los siguientes propósitos: a) describir los desempeños en las competencias motrices de control de objetos y control del cuerpo de estudiantes de primer y segundo grado de primaria de la Región de La Araucanía, Chile; b) determinar la relación entre la competencia motriz y la edad; y c) establecer la existencia de diferencias según el sexo de los estudiantes de la Región de La Araucanía, Chile.

\section{METODOLOGÍA}

La presente investigación aborda la problemática de la competencia motriz en escolares chilenos desde un enfoque cuantitativo, siendo un estudio no experimental de corte transversal y de tipo descriptivo-correlacional.

\section{Participantes}

La muestra no probabilística incidental estuvo compuesta por 289 estudiantes $(51.2 \%$ niñas, $48.8 \%$ niños) de entre seis y ocho años (edad $\mathrm{M}=6.98 \mathrm{DE}=0.68$ ) de primer y segundo grado de primaria de cinco Centros Educacionales de la región de La Araucanía, Chile. Los criterios de inclusión fueron: a) estar en primer o segundo grado de primaria; b) participar regularmente de las clases de Educación Física; c) estar sano y no haber recibido diagnóstico de discapacidad física o intelectual con carácter previo al estudio. Se excluyeron a los participantes que no completaron todas las tareas del test. El protocolo de investigación contó con la aprobación del Departamento de Educación Municipal de Temuco. El estudio se realizó siguiendo las normas y los principios éticos de la Declaración de Helsinki para la investigación 
con seres humanos. Se solicitó a los padres y apoderados su consentimiento por escrito, mientras que a los estudiantes se les informó que la participación del estudio era voluntaria por lo que podían decidir no participar si así lo manifestaban.

\section{Materiales e Instrumento}

Para la evaluación de la competencia motriz se aplicó el test MOBAK 1-2 de Herrmann, Gerlach, y Seelig (2015), cuya versión en español fue validada por Carcamo-Oyarzun y Herrmann (2020). La Batería MOBAK (acrónimo de Motorische Basiskompetenzen en alemán) se enfoca en la funcionalidad, es decir, en la solución de las tareas motrices, dividiéndose en diversos tramos según el grado que los escolares estén cursando, y cuyas tareas se vinculan con los objetivos del currículum (Carcamo-Oyarzun, Peña-Troncoso, y Cumilef-Bustamante, en prensa; Herrmann et al.,_2015). Es así como el test MOBAK 1-2 ha sido diseñado para escolares de primer y segundo grado de primaria, estableciéndose su pertinencia con el currículum chileno de Educación Física a través de la respectiva validez de contenido (Carcamo-Oyarzun et al., en prensa).

El test MOBAK 1-2 cuenta con una estructura dividida en dos dimensiones (constructos latentes) denominados Control de Objetos (CO) y Control del Cuerpo (CC), las que agrupan cuatro tareas motrices (ítems observables) por dimensión. Esta estructura de dos dimensiones compuestas por cuatro tareas cada una fue confirmada factorialmente (Carcamo-Oyarzun y Herrmann, 2020), en donde el modelo presentó adecuados índices de ajuste ( $x^{2}=34.29$; $d f=19 ; p=0.017 ; C F I=0.962 ; R M S E A=0.038$ ). Los ítems que conforman la dimensión de $\mathrm{CO}$ se vinculan a las habilidades motrices de manipulación, mientras que la dimensión de CC está compuesta por tareas motrices relacionadas con habilidades de estabilidad y habilidades de locomoción. Las tareas para el test MOBAK 1-2 se presentan en la tabla 1. 
Tabla 1

Descripción de las tareas motrices del test MOBAK 1-2

\begin{tabular}{|c|c|c|}
\hline Dimensiones & Ítems & Descripción \\
\hline \multirow{4}{*}{$\begin{array}{l}\text { Control de Objetos } \\
\text { (CO) }\end{array}$} & Lanzar & $\begin{array}{l}\text { Lanzar una pelota para tratar de acertar a un } \\
\text { círculo marcado en la pared, desde } 2.0 \text { mts. de } \\
\text { distancia. }\end{array}$ \\
\hline & Atrapar & $\begin{array}{l}\text { Atrapar una pelota de goma después de un } \\
\text { rebote en el piso. }\end{array}$ \\
\hline & Conducir con la mano & $\begin{array}{l}\text { Conducir con la mano una pelota de básquetbol } \\
\mathrm{N}^{\circ} 3 \text { por un carril }(5.0 \mathrm{mts} \text {. } \times 1.0 \mathrm{mt} \text {.) }\end{array}$ \\
\hline & Conducir con el pie & $\begin{array}{l}\text { Conducir con el pie una pelota de futsal } \mathrm{N}^{\circ} 4 \text { por } \\
\text { un carril (5.0 mts. } \times 1.0 \mathrm{mt} \text {.). }\end{array}$ \\
\hline \multirow{4}{*}{$\begin{array}{l}\text { Control del Cuerpo } \\
\text { (CC) }\end{array}$} & Equilibrio & $\begin{array}{l}\text { Caminar hacia adelante sobre una banca } \\
\text { invertida que se balancea. }\end{array}$ \\
\hline & Rodar & $\begin{array}{l}\text { Realizar una voltereta hacia adelante, partiendo } \\
\text { en cuclillas con las manos apoyadas en la } \\
\text { colchoneta. }\end{array}$ \\
\hline & Saltar & $\begin{array}{l}\text { Saltar de forma continua } 4 \text { cuadrados } \\
\text { instalados en el piso, apoyando } 1 \text { pie entre los } \\
\text { cuadrados y los } 2 \text { pies al lado de los cuadrados. }\end{array}$ \\
\hline & Correr & $\begin{array}{l}\text { Correr de forma lateral sobre una línea de } 3.0 \\
\text { mts. }\end{array}$ \\
\hline
\end{tabular}

Fuente: elaboración propia con base en Carcamo-Oyarzun y Herrmann (2020).

Para cada una de las tareas motrices, los estudiantes tienen dos intentos, excepto en las tareas de atrapar y lanzar, en donde los intentos son seis. Para las tareas que constan de 2 intentos, la puntuación se realiza de siguiente forma: tarea nunca lograda $=0$ punto; tarea una vez lograda=1 punto; tarea dos veces lograda=2 puntos, mientras que para las tareas de seis intentos (atrapar y lanzar) la puntuación es de la siguiente manera: tarea lograda 0-2 veces $=0$ punto; tarea lograda $3-4$ veces $=1$ punto; y tarea lograda $5-6$ veces $=2$ puntos. Teniendo en cuenta de que cada ítem puede ser valorado con un mínimo de cero puntos y un máximo de dos puntos, y que cada dimensión consta de cuatro ítems, el máximo de puntaje a alcanzar en cada dimensión (Control de Objetos - Control del Cuerpo) es de ocho puntos (2 puntos $\times 4$ tareas). Los escolares que obtengan entre cero y dos puntos (de un máximo de ocho) en cada dimensión presentarían necesidad de reforzamiento motriz; los escolares que obtengan entre tres y seis puntos estarían alcanzando parcialmente los objetivos planteados en el curriculum; mientras que los escolares que alcanzan siete y ocho puntos estarían cumpliendo ampliamente con los objetivos curriculares (Herrmann, 2018, p. 40-41). Para una revisión detallada de los procedimientos para la aplicación y valoración del test MOBAK 1-2 véase el manual de aplicación en español, disponible en www.mobak.cl. 


\section{Procedimiento}

Se realizaron reuniones con los directores de los Centros Educativos y profesores de Educación Física, para explicarles los objetivos del estudio y la metodología a utilizar, así como para coordinar las fechas y horarios de aplicación del Test MOBAK 1-2.

EI Test MOBAK 1-2 fue aplicado por un equipo evaluador de ocho personas capacitadas y con experiencia en la aplicación de los tramos de la batería MOBAK para educación primaria, en los horarios en donde el estudiantado tenía clases de Educación Física. Antes del inicio de la clase y del arribo del estudiantado, el equipo evaluador realizaba la marcación e instalación de las estaciones. Cada clase se dividía en ocho grupos (de entre tres a cinco estudiantes cada uno, dependiendo del tamaño de la clase). Cada grupo era dirigido por un evaluador o evaluadora, quien los llevaba a cada una de las estaciones para proceder con las evaluaciones. En cada estación el evaluador o evaluadora explicaba cuál era la tarea motriz a realizar y luego la demostraba. Cada niño o niña realizaba dos intentos (a excepción de las tareas de lanzar y atrapar, en donde realizaban seis), sin permitirse intentos de prueba. La duración aproximada de la aplicación total del test fue de 45 minutos.

\section{Análisis estadístico}

Los datos fueron analizados con el programa SPSS Statistics 25.0. Se llevaron a cabo análisis descriptivos usando medidas de tendencia central y dispersión. Se analizó la normalidad de la muestra a través de la prueba de Kolmogorov Smirnov. Se comprobó que la muestra no cumple el principio de normalidad, por lo que decidió utilizar pruebas de estadística no paramétricas. De esta manera, se realizaron análisis mediante el coeficiente rho de Spearman para establecer la relación entre la competencia motriz y la edad, y se llevaron a cabo análisis diferenciales utilizando la prueba $U$ de Mann-Whitney para muestras independientes, para determinar la existencia de diferencias según el sexo. El tamaño del efecto fue determinado a través del cálculo de la probabilidad de superioridad ( $P S_{\text {est }}$ ), procedimiento recomendado para comparaciones de dos grupos a través de pruebas no paramétricas, interpretándose $P S_{\text {est }} \geq 0.56$ como un tamaño de efecto pequeño, $P S_{\text {est }} \geq 0.64$ mediano, y $\mathrm{PS}_{\text {est }} \geq 0.71$ grande (Grissom, 1994).

\section{RESULTADOS}

En la tabla 2 se presentan los análisis descriptivos por sexo (media, desviación estándar e intervalo de confianza al 95\% [IC95\%]). Al observar las medias de los desempeños en función del sexo, es posible identificar que, de las tareas motrices relacionadas con el $\mathrm{CO}$, la de mayor dificultad fue Lanzar, tanto para las niñas $(M=0.49)$ como para los niños $(M=0.79)$. En la dimensión de CC, la tarea motriz en donde ambos sexos demostraron el menor desempeño fue Saltar (niñas $M=0.84$, niños $M=0.71$ ). Por el contrario, la tarea en donde se 
observó que niños y niñas obtuvieron un mejor desempeño en la dimensión de CO fue en el ítem Atrapar (niñas $M=1.21$, niños $M=1.32$ ), mientras que en la dimensión $C C$, la tarea Correr fue en donde demostraron el mejor desempeño (niñas $M=1.36$, niños $M=1.45$ ).

Tabla 2

Estadísticos Descriptivos de los ítems del test MOBAK 1-2 en función del sexo.

\begin{tabular}{|c|c|c|c|c|c|c|}
\hline \multirow{2}{*}{ Ítem } & \multicolumn{2}{|c|}{ Niñas } & \multicolumn{2}{|c|}{ Niños } & \multicolumn{2}{|c|}{ Total } \\
\hline & $M(D E)$ & IC 95\% & $M(D E)$ & IC 95\% & M (DE) & IC 95\% \\
\hline Lanzar & $0.49(0.64)$ & {$[0.38-0.59]$} & $0.79(0.73)$ & {$[0.67-0.91]$} & $0.63(070)$ & {$[0.55-0.71]$} \\
\hline Atrapar & $1.21(0.82)$ & [1.08-1.34] & $1.32(0.74)$ & {$[1.20-1.44]$} & $1.26(0.78)$ & {$[1.17-1.35]$} \\
\hline $\begin{array}{l}\text { Conducir con } \\
\text { la mano }\end{array}$ & $0.55(0.77)$ & {$[0.42-0.67]$} & $1.04(0.91)$ & [0.88-1.19] & $0.79(0.87)$ & [0.68-0.89] \\
\hline $\begin{array}{c}\text { Conducir con } \\
\text { el pie }\end{array}$ & $1.01(0.83)$ & [0.88-1.15] & $1.14(0.78)$ & [1.01-1.27] & $1.08(0.81)$ & [0.98-1.17] \\
\hline $\begin{array}{c}\text { Dimensión } \\
\text { Control } \\
\text { de Objetos }\end{array}$ & $3.26(1.89)$ & [2.95-3.56] & $4.28(2.01)$ & [3.95-4.62] & $3.76(2.01)$ & [3.53-3.99] \\
\hline Equilibrio & $1.30(0.89)$ & {$[1.16-1.45]$} & $1.34(0.89)$ & [1.19-1.49] & $1.32(0.88)$ & [1.22-1.42] \\
\hline Rodar & $1.22(0.88)$ & {$[1.07-1.36]$} & $1.33(0.85)$ & [1.18-1.47] & $1.27(0.86)$ & [1.17-1.37] \\
\hline Saltar & $0.84(0.84)$ & {$[0.70-0.97]$} & $0.71(0.80)$ & [0.58-0.84] & $0.78(0.82)$ & [0.68-0.87] \\
\hline Correr & $1.36(0.82)$ & [1.23-1.49] & $1.45(0.79)$ & [1.32-1.59] & $1.40(0.80)$ & [1.31-1.50] \\
\hline $\begin{array}{l}\text { Dimensión } \\
\text { Control } \\
\text { del Cuerpo }\end{array}$ & $4.72(2.09)$ & {$[4.38-5.06]$} & $4.83(2.16)$ & {$[4.47-5.19]$} & $4.77(2.12)$ & [4.53-5.02] \\
\hline
\end{tabular}

Fuente: elaboración propia.

En la tabla 3 se presentan las correlaciones entre los ítems del test MOBAK 1-2 correspondiente a las dimensiones de CO y CC, y la edad. De manera global, es posible observar que tanto la dimensión $C O(p<0.001$; rho=0.32) como la dimensión $C C(p<0.001$; rho $=0.16$ ) se relacionan positivamente con la edad. 
Tabla 3

Correlación entre los ítems del test MOBAK 1-2, las dimensiones Control de Objetos y Control del Cuerpo, y la edad.

\begin{tabular}{|c|c|c|c|c|c|c|c|c|c|}
\hline Ítem & 1 & 2 & 3 & 4 & 5 & 6 & 7 & 8 & Edad \\
\hline 1. Lanzar & - & - & - & - & - & - & - & - & $0.253^{* *}$ \\
\hline 2. Atrapar & $0.199^{\star \star}$ & - & - & - & - & - & - & - & $0.320^{* *}$ \\
\hline $\begin{array}{l}\text { 3. Conducir } \\
\text { con la mano }\end{array}$ & $0.321^{* *}$ & $0.275^{\star *}$ & - & - & - & - & - & - & $0.244^{\star *}$ \\
\hline $\begin{array}{l}\text { 4. Conducir } \\
\text { con el pie }\end{array}$ & $0.130^{*}$ & $0.146^{*}$ & $0.145^{*}$ & - & - & - & - & - & 0.005 \\
\hline 5. Equilibrio & $0.134^{*}$ & $0.149^{*}$ & $0.194^{* *}$ & $0.210^{* *}$ & - & - & - & - & 0.059 \\
\hline 6. Rodar & $0.136^{*}$ & $0.165^{* *}$ & $0.256^{* *}$ & $0.148^{*}$ & $0.281^{* *}$ & - & - & - & 0.084 \\
\hline 7. Saltar & $0.133^{*}$ & $0.186^{* *}$ & 0.099 & $0.148^{*}$ & $0.167^{* *}$ & 0.101 & - & - & $0.131^{*}$ \\
\hline 8. Correr & $0.200^{* *}$ & $0.154^{* *}$ & $0.214^{* *}$ & $0.237^{* *}$ & $0.156^{* *}$ & $0.172^{* *}$ & $0.269^{* *}$ & - & 0.114 \\
\hline $\begin{array}{l}\text { Dimensión } \\
\text { Control } \\
\text { de Objetos }\end{array}$ & $0.599^{* *}$ & $0.635^{* *}$ & $0.712^{* *}$ & $0.560^{* *}$ & $0.280^{* *}$ & $0.281^{* *}$ & $0.216^{* *}$ & $0.320^{* *}$ & $0.315^{* *}$ \\
\hline $\begin{array}{l}\text { Dimensión } \\
\text { Control } \\
\text { del Cuerpo }\end{array}$ & $0.237^{* *}$ & $0.271^{* *}$ & $0.310^{* *}$ & $0.306^{* \star}$ & $0.640^{* *}$ & $0.613^{* *}$ & $0.618^{* *}$ & $0.607^{* *}$ & $0.161^{* *}$ \\
\hline
\end{tabular}

Nota. ${ }^{*}$ Correlación significativa en el nivel $0.05 .{ }^{* *}$ Correlación significativa en el nivel 0.001 . Fuente: elaboración propia.

En la tabla 4 se presentan los resultados de la comparación del desempeño motriz en función del sexo, tanto para cada tarea como para cada dimensión. Al realizar un análisis por tarea motriz, sólo en Lanzar $\left(p<0.001, \mathrm{PS}_{\text {est }}=0.57\right)$ y en Conducir con la mano $(p<0.001$, $P S_{\text {est }}=0.60$ ) se encontraron diferencias estadísticamente significativas, ambos con un tamaño del efecto pequeño, en donde los niños presentan mejores desempeños que las niñas. El análisis global indica que existen diferencias estadísticamente significativas en la dimensión de $\mathrm{CO}$, en donde los niños presentan un mayor rendimiento en comparación a las niñas $(p<0.001)$ con un tamaño de efecto pequeño $\left(P S_{\text {est }}=0.61\right)$; mientras que en la dimensión de $C C$ no se encontraron diferencias estadísticamente significativas $(p=0.522)$. 
Tabla 4

Diferencias en las tareas motrices de Control de Objetos (CO) y Control del Cuerpo (CC) según sexo.

\begin{tabular}{|c|c|c|c|c|c|c|c|}
\hline Tarea/Dimensión & Sexo & Mdn & rangos & U & Z & $\mathrm{p}$ & $\mathrm{PS}_{\text {est }}$ \\
\hline \multirow{2}{*}{ Lanzar } & niña & 0 & 129.00 & \multirow{2}{*}{12802.000} & \multirow{2}{*}{-3.675} & \multirow{2}{*}{$0.000^{* *}$} & \multirow{2}{*}{0.57} \\
\hline & niño & 1 & 161.79 & & & & \\
\hline \multirow{2}{*}{ Atrapar } & niña & 1 & 140.48 & \multirow{2}{*}{11102.500} & \multirow{2}{*}{-1.019} & \multirow{2}{*}{0.308} & \multirow{2}{*}{-} \\
\hline & niño & 1 & 149.74 & & & & \\
\hline \multirow{2}{*}{$\begin{array}{l}\text { Conducir con la } \\
\text { mano }\end{array}$} & niña & 0 & 124.59 & \multirow{2}{*}{13455.000} & \multirow{2}{*}{-4.655} & \multirow{2}{*}{$0.000^{* *}$} & \multirow{2}{*}{0.60} \\
\hline & niño & 1 & 166.43 & & & & \\
\hline \multirow{2}{*}{ Conducir con el pie } & niña & 1 & 139.10 & \multirow{2}{*}{11307.000} & \multirow{2}{*}{-1.306} & \multirow{2}{*}{0.192} & \multirow{2}{*}{-} \\
\hline & niño & 1 & 151.19 & & & & \\
\hline \multirow{2}{*}{$\begin{array}{l}\text { Total Dimensión } \\
\text { Control de Objetos }\end{array}$} & niña & 3 & 123.93 & \multirow{2}{*}{13553.000} & \multirow{2}{*}{-4.439} & \multirow{2}{*}{$0.000^{* *}$} & \multirow{2}{*}{0.61} \\
\hline & niño & 4 & 167.12 & & & & \\
\hline \multirow{2}{*}{ Equilibrio } & niña & 2 & 143.32 & \multirow{2}{*}{10683.000} & \multirow{2}{*}{-.403} & \multirow{2}{*}{0.687} & \multirow{2}{*}{-} \\
\hline & niño & 2 & 146.77 & & & & \\
\hline \multirow{2}{*}{ Rodar } & niña & 2 & 140.30 & \multirow{2}{*}{11130.000} & \multirow{2}{*}{-1.087} & \multirow{2}{*}{0.277} & \multirow{2}{*}{-} \\
\hline & niño & 2 & 149.94 & & & & \\
\hline Saltar & niña & 1 & 150.68 & 9593.000 & -1279 & 0.201 & - \\
\hline & niño & 0 & 139.04 & & - 1.210 & & - \\
\hline Correr & niña & 2 & 140.51 & 11098500 & -1072 & 0284 & - \\
\hline correr & niño & 2 & 149.71 & 11050.000 & -1.072 & 0.204 & - \\
\hline Total Dimensión & niña & 5 & 141.97 & 10883000 & & & \\
\hline Control del Cuerpo & niño & 5 & 148.18 & 10000.000 & -.040 & $0.0<2$ & - \\
\hline
\end{tabular}

Nota. ${ }^{* *}$ Diferencia significativa en el nivel 0.001. Niñas $n=148$, Niños $n=141$. Mdn $=$ mediana.

Fuente: elaborada por los autores.

\section{DISCUSIÓN}

Considerando que la competencia motriz es un agente relevante para la participación en actividades relacionadas con el juego activo y con los deportes, y que se asocian positivamente con la práctica regular de actividad física, este estudio ha tenido como objetivos describir el desempeño en la competencia motriz de control de objetos y control del cuerpo de estudiantes de primer y segundo grado de primaria de Chile; determinar la relación entre la competencia motriz y la edad; y establecer la existencia de posibles diferencias según el sexo del estudiantado.

Los resultados indican que los estudiantes que participaron de este estudio presentan valores más altos en CC que en CO, coincidiendo con los hallazgos de Foulkes et al. (2015). Al contrastar de manera descriptiva estos resultados con otras investigaciones internacionales que también han utilizado el test MOBAK 1-2, es posible notar que el desempeño de los estudiantes chilenos difiere dependiendo de la dimensión de competencia motriz. Es así como en la dimensión de $\mathrm{CO}$, los estudiantes de este estudio presentaron 
valores más bajos que los participantes en estudios realizados en Alemania (Herrmann et al., 2019), Suiza (Herrmann et al., 2015) y Portugal (Quitério et al., 2018). En lo que respecta a la dimensión de CC los estudiantes de este trabajo obtienen resultados superiores a los estudiantes alemanes que participaron del estudio de Herrmann et al. (2019), similares a la muestra de estudiantes portugueses del estudio de Quitério et al. (2018), e inferiores a los estudiantes suizos participantes del estudio de Herrmann et al. (2015).

En cuanto a la relación entre la competencia motriz y la edad de los y las estudiantes, los resultados arrojaron asociaciones tanto para $\mathrm{CO}$ como para $\mathrm{CC}$, coincidiendo con las conclusiones de la revisión sistemática de Barnett et al. (2016), señalándose que el desempeño motriz se relaciona positivamente con la edad, en donde a medida que ésta se incrementa se van presentando mejores desempeños. Teniendo en cuenta que la competencia motriz no se adquiere por sí sola con el paso del tiempo, la asociación positiva entre el incremento de la edad y el desempeño motriz no tan solo se debería al crecimiento, sino que también a la práctica y a la interacción con el medio ambiente. Haywood y Getchell (2019) indican que, desde una perspectiva ecológica, a edades mayores se evidenciaría un mejor dominio motriz debido tanto por factores de maduración y de desarrollo, así como también por las experiencias adquiridas al interactuar con su entorno. De esta forma, es posible que la asociación positiva entre la edad y la competencia motriz de los participantes de este estudio se deba a que los estudiantes de segundo año de primaria han tenido mayores experiencias motrices que los niños y niñas de primer año de primaria.

En lo que respecta a las diferencias según el sexo de los estudiantes, al comparar los desempeños de ambos sexos se encontraron diferencias estadísticamente significativas en la dimensión de $\mathrm{CO}$, en donde los niños presentan mejores rendimientos que las niñas; mientras que en el CC no se encontraron diferencias significativas entre niños y niñas. Diversos estudios confirman que el sexo es un correlato de las actividades vinculadas a la manipulación de objetos, en donde los niños presentarían mejores desempeños que las niñas (Barnett et al.,_2016; Herrmann et al.,_2019); sin embargo, este consenso no existe en lo que respecta a las tareas motrices de CC. Por un lado, los resultados de este estudio coinciden con las conclusiones de Barnett et al. (2016), quienes no encontraron evidencia de que el sexo de los estudiantes se asocie con tareas vinculadas al CC. Por el contrario, estos resultados difieren con los de livonen y Sääkslahti (2014), quienes sí encontraron evidencias de que existen relaciones significativas entre sexo y el CC. Estos resultados dispares dejan de manifiesto la heterogeneidad de resultados que han abordado la relación de las tareas motrices vinculadas a la locomoción y estabilidad con el sexo de los estudiantes.

Según lo anteriormente expuesto, se evidencia que no todos los estudiantes chilenos de primer y segundo de primaria pueden cumplir de manera satisfactoria las tareas motrices vinculadas al control de objetos y el control del cuerpo, por lo que la Educación Física aparece 
como una instancia esencial para poder revertir dicha situación, ya que uno de sus objetivos principales es el desarrollo de las habilidades motrices, que les permitan a los estudiantes no tan solo desenvolverse de manera eficiente en su entorno, sino que también para fomentar en ellos la adquisición de un estilo de vida activo y saludable (Ministerio de Educación, 2013). Por esa razón es importante la evaluación y monitoreo permanente del cumplimiento de estos objetivos estipulados en el currículum, ya que la identificación del nivel de desempeño permitirá al profesorado reflexionar sobre las debilidades o fortalezas del alumnado, para así crear estrategias pedagógicas que busquen satisfacer las necesidades de reforzamiento motriz de sus estudiantes, de tal forma que éstas se transformen en una instancia acorde a las necesidades del estudiantado y fomenten el desarrollo equitativo de la competencia motriz. Considerando que el alumnado percibe que el profesorado de Educación Física conoce lo que puede (o no) realizar (Cárcamo, 2012), se debe confirmar dicha percepción elaborando actividades donde todos y todas puedan desempeñarse de acuerdo a sus posibilidades. Tal como lo plantea Brito (2006), para edificar la competencia motriz es necesario poner a los estudiantes en situaciones didácticas que les permitan alcanzar objetivos, resolver problemas y tomar decisiones. Para poder generar estas situacionesproblema, previamente se debe saber si el estudiantado será capaz de resolverlas, por lo que, si se desconocen las potencialidades de nuestros estudiantes, se corre el riesgo de plantear actividades cuya dificultad sobrepase las competencias del estudiantado, impidiéndoles solucionar el problema motriz y generando un sentimiento de frustración e incompetencia (Ruiz Perez, 2018).

De esta forma, se recomienda generar situaciones didácticas en base a las experiencias y aprendizajes previos del estudiantado, para desde ahí construir ambientes y tareas que los enfrenten a situaciones problema que consideren la variabilidad de la práctica (Brito, 2006). Así, al plantear una situación que el estudiantado pueda resolver de manera competente, es necesario continuar presentando tareas que vayan variando las condiciones tanto en espacio, material, tiempo, relaciones, entre otros (Scheuer y Heck, 2020), reinventando lo conocido, lo vivido, y lo comprendido para solucionar la situación original, para luego superar y resolver una segunda situación, movilizando los aprendizajes previos y enriqueciendo la competencia motriz (Brito, 2006).

Los resultados del presente estudio aportan información relevante para el profesorado de Educación Física, para conocer el desempeño motriz de los escolares y el logro de los aprendizajes alcanzados en la asignatura de Educación Física. Teniendo en cuenta de que existe muy poca evidencia del rendimiento motriz en la población escolar (Pavez-Adasme et al., 2020), se recomienda que futuras investigaciones continúen entregando información sobre la competencia motriz de escolares de enseñanza primaria, para poder generar normas estandarizadas que establezcan diferentes niveles de desempeño, permitiendo detectar a 
estudiantes que necesiten de un reforzamiento motriz o, por otro lado, para identificar a niños y niñas con desempeños destacados como potenciales talentos deportivos. Además, se sugiere incorporar otras variables que emergen como correlatos subyacentes entre la competencia motriz y la actividad física, como la percepción de competencia motriz (Carcamo-Oyarzun, Estevan, y Herrmann, 2020; De Messter, et al., 2020), motivación (Bardid, et al., 2016; De Meester et al., 2016) y otras variables volitivas que permitan entender cómo el desarrollo de la competencia motriz puede fomentar la práctica regular de actividad física en la población escolar.

\section{REFERENCIAS}

Bardid, F., de Meester, A., Tallir, I., Cardon, G., Lenoir, M., y Haerens, L. (2016). Configurations of actual and perceived motor competence among children: Associations with motivation for sports and global self-worth. Human Movement Science, 50, 1-9. doi: https://doi.org/10.1016/j.humov.2016.09.001

Barnett, L. M., Lai, S. K., Veldman, S. L. C., Hardy, L. L., Cliff, D. P., Morgan, P. J., Zask, A., ... Okely, A. D. (2016). Correlates of Gross Motor Competence in Children and Adolescents: A Systematic Review and Meta-Analysis. Sports Medicine, 46(11), 16631688. doi: https://doi.org/10.1007/s40279-016-0495-z

Brito, L. (2006). Edificación de la competencia motriz y conquista de las realizaciones personales. Lúdica Pedagógica, 2(11), 96-107. doi: https://doi.org/10.17227/ludica.num11-7657

Cairney, J., Dudley, D., Kwan, M., Bulten, R., y Kriellaars, D. (2019). Physical Literacy, Physical Activity and Health: Toward an Evidence-Informed Conceptual Model. Sports Medicine, 49(3), 371-383. doi: https://doi.org/10.1007/s40279-019-01063-3

Carcamo, J. (2012). El profesor de Educación Física desde la perspectiva de los escolares. Estudios Pedagógicos (Valdivia), 38(1), 105-119. doi: https://doi.org/10.4067/S071807052012000100006

Carcamo-Oyarzun, J., y Herrmann, C. (2020). Validez de constructo de la batería MOBAK para la evaluación de las competencias motrices básicas en escolares de educación primaria. Revista Española De Pedagogía, 78(276), 291-308. doi: https://doi.org/10.22550/REP78-2-2020-03

Carcamo-Oyarzun, J., Estevan, I., y Herrmann, C. (2020). Association between Actual and Perceived Motor Competence in School Children. International Journal of Environmental Research and Public Health, 17(10). doi: https://doi.org/10.3390/ijerph17103408

Carcamo-Oyarzun, J., Peña-Troncoso, S., y Cumilef-Bustamante, P. (en prensa). Validez de contenido de la Batería MOBAK para la evaluación del eje curricular de habilidades motrices en Educación Física. Estudios Pedagógicos, 50(1). 
De Meester, A., Barnett, L. M., Brian, A., Bowe, S. J., Jiménez-Díaz, J., van Duyse, F., ... Haerens, L. (2020). The Relationship Between Actual and Perceived Motor Competence in Children, Adolescents and Young Adults: A Systematic Review and Meta-analysis. Sports Medicine, 50(11), 2001-2049. doi: https://doi.org/10.1007/s40279-020-01336-2 De Meester, Maes, J., Stodden, D., Cardon, G., Goodway, J., Lenoir, M., y Haerens, L. (2016). Identifying profiles of actual and perceived motor competence among adolescents: Associations with motivation, physical activity, and sports participation. Journal of Sports Sciences, 34(21), 2027-2037. doi: https://doi.org/10.1080/02640414.2016.1149608

Dyson, B. (2014). Quality physical education: A commentary on effective physical education teaching. Research Quarterly for Exercise and Sport, 85(2), 144-152. doi: https://doi.org/10.1080/02701367.2014.904155

Ennis, C. D. (2011). Physical Education Curriculum Priorities: Evidence for Education and Skillfulness. Quest, 63(1), 5-18. doi: https://doi.org/10.1080/00336297.2011.10483659

Foulkes, J. D., Knowles, Z., Fairclough, S. J., Stratton, G., O'Dwyer, M., Ridgers, N. D., y Foweather, L. (2015). Fundamental Movement Skills of Preschool Children in Northwest England. Perceptual and Motor Skills, 121(1), 260-283. doi: https://doi.org/10.2466/10.25.PMS.121c14x0

Gallahue, D. L., Ozmun, J. C., y Goodway, J. D. (2012). Understanding motor development: Infants, children, adolescents, adults (7th ed.). New York: McGraw-Hill.

Gerlach, E., Herrmann, C., Jekauc, D., y Wagner, M. O. (2017). Diagnostik motorischer Leistungsdispositionen. In U. Trautwein y M. Hasselhorn (Eds.), Begabungen und Talente (Jahrbuch der pädagogisch-psychologischen Diagnostik, Tests \& Trends) (pp. 145-158). Göttingen: Hogrefe.

Grissom, R. J. (1994). Probability of the superior outcome of one treatment over another. Journal of Applied Psychology, 79(2), 314-316. doi: https://doi.org/10.1037/0021$\underline{9010.79 .2 .314}$

Hardy, L. L., Reinten-Reynolds, T., Espinel, P., Zask, A., y Okely, A. D. (2012). Prevalence and correlates of low fundamental movement skill competency in children. Pediatrics, 130(2), e390-398. doi: https://doi.org/10.1542/peds.2012-0345

Haywood, K., y Getchell, N. (2019). Life span motor development ( $7^{\text {th }}$ ed.). Champaign IL: Human Kinetics.

Herrmann, C. (2018). MOBAK 1-4: Test zur Erfassung Motorischer Basiskompetenzen für die Klassen 1-4. Göttingen: Hogrefe.

Herrmann, C., Gerlach, E., y Seelig, H. (2015). Development and Validation of a Test Instrument for the Assessment of Basic Motor Competencies in Primary School. Measurement in Physical Education and Exercise Science, 19(2), 80-90. doi: https://doi.org/10.1080/1091367X.2014.998821 
Herrmann, C., Heim, C., y Seelig, H. (2019). Construct and correlates of basic motor competencies in primary school-aged children. Journal of Sport and Health Science, 8(1), 63-70. doi: https://doi.org/10.1016/j.jshs.2017.04.002

Holfelder, B., y Schott, N. (2014). Relationship of fundamental movement skills and physical activity in children and adolescents: A systematic review. Psychology of Sport and Exercise, 15(4), 382-391. doi: https://doi.org/10.1016/j.psychsport.2014.03.005

livonen, S., y Sääkslahti, A. K. (2014). Preschool Children's Fundamental Motor Skills: A Review of Significant Determinants. Early Child Development and Care, 184(7), 11071126. Recuperado de https://eric.ed.gov/?id=EJ1030457

Jiménez-Díaz, J., Salazar-Rojas, W., y Morera, M. (2015). Diferencias en el desempeño de los patrones básicos de movimiento según la edad y el sexo (versión traducida al español). Pensar En Movimiento: Revista de Ciencias del Ejercicio y La Salud, 13(2), 17-33. doi: https://doi.org/10.15517/PENSARMOV.V13I2.22024

Logan, S. W., Kipling, E., Getchell, N., Pfeiffer, K. A., y Robinson, L. E. (2015). Relationship Between Fundamental Motor Skill Competence and Physical Activity During Childhood and Adolescence: A Systematic Review. Kinesiology Review, 4(4), 416-426. doi: https://doi.org/10.1123/kr.2013-0012

Logan, S. W., Ross, S. M., Chee, K., Stodden, D. F., y Robinson, L. E. (2018). Fundamental motor skills: A systematic review of terminology. Journal of Sports Sciences, 36(7), 781796. doi: https://doi.org/10.1080/02640414.2017.1340660

Lopes, L., Santos, R., Coelho-E-Silva, M., Draper, C., Mota, J., Jidovtseff, B., . . AgostinisSobrinho, C. (2021). A Narrative Review of Motor Competence in Children and Adolescents: What We Know and What We Need to Find Out. International Journal of Environmental Research and Public Health, 18(1). doi: https://doi.org/10.3390/ijerph18010018

Ministerio de Educación (2013). Bases Curriculares Educación Física y Salud de $1^{\circ}$ a $6^{\circ}$ Básico. Santiago de Chile: Gobierno de Chile.

Morgan, P. J., Barnett, L. M., Cliff, D. P., Okely, A. D., Scott, H. A., Cohen, K. E., y Lubans, D. R. (2013). Fundamental movement skill interventions in youth: A systematic review and meta-analysis. Pediatrics, 132(5), e1361-e1383. doi: https://doi.org/10.1542/peds.20131167

Pavez-Adasme, G., Hernández-Mosqueira, C., Torres, S., Paillacar, M., Concha, C., Cabrera, M., Concha, M., y Gómez-Álvarez, N. (2020). Test de desarrollo motor aplicados en Chile entre el período 2014-2018. Una revisión sistemática. Revista Ciencias de la Actividad Física UCM, 21(1), 1-13. doi: https://doi.org/10.29035/rcaf.21.1.1

Quitério, A., Martins, J., Onofre, M., Costa, J., Mota, J., Gerlach, E., Scheur, C. y Herrmann, C. (2018). Mobak 1 Assessment in Primary Physical Education: Exploring Basic Motor 
Competences of Portuguese 6-Year-Olds. Perceptual and Motor Skills, 125(6), 10551069. doi: https://doi.org/10.1177/0031512518804358

Robinson, L. E., Stodden, D. F., Barnett, L. M., Lopes, V. P., Logan, S. W., Rodrigues, L. P., y D'Hondt, E. (2015). Motor Competence and its Effect on Positive Developmental Trajectories of Health. Sports Medicine, 45(9), 1273-1284. doi: https://doi.org/10.1007/s40279-015-0351-6

Ruiz Perez, L. M. (1995). Competencia motriz: Elementos para comprender el aprendizaje motor en educación física escolar. Madrid: Gymnos.

Ruiz Perez, L. M. (2018). Low Competence and Developmental Motor Coordination Problems in Physical Education. RICYDE. Revista Internacional De Ciencias Del Deporte, 14(52), 97-100. doi: https://doi.org/10.5232/ricyde2018.052ed

Scheuer, C., y Heck, S. (2020). Toolkit für die Fortbildung von Sportlehrkräften. Luxemburgo: Université du Luxembourg. Retrieved from https://orbilu.uni.lu/handle/10993/43043

Scheuer, C., Herrmann, C., y Bund, A. (2019). Motor tests for primary school aged children: A systematic review. Journal of Sports Sciences, 37(10), 1097-1112. doi: https://doi.org/10.1080/02640414.2018.1544535

Stodden, D. F., Goodway, J. D., Langendorfer, S. J., Roberton, M. A., Rudisill, M. E., Garcia, C., y Garcia, L. E. (2008). A Developmental Perspective on the Role of Motor Skill Competence in Physical Activity: An Emergent Relationship. Quest, 60(2), 290-306. doi: https://doi.org/10.1080/00336297.2008.10483582

Ulrich, D. A. (1985). Test of gross motor development. Austin, TX: Pro-Ed. Recuperado de https://www.researchgate.net/publication/283529890 TGMD Test of Gross Motor D evelopment 